\title{
LAS PUBLICACIONES COMERCIALES (TRADE JOURNALS), FUENTES FUNDAMENTALES PARA LAS HISTORIAS AZUCARERAS DEL CARIBE: LE JOURNAL DES FABRICANTS DE SUCRE, THE SUGAR CANE Y THE LOUISIANA PLANTER AND SUGAR MANUFACTURER
}

\author{
Humberto García Muñiz \\ Instituto de Estudios del Caribe, Universidad de Puerto Rico, Recinto de Río Piedras \\ hgarciamuniz@gmail.com
}

Recibido: 10 julio 2014. Aceptado: 1 febrero 2015.

Cómo citar este artículo/Citation: García Muñiz, Humberto (2015), "Las Publicaciones Comerciales (Trade Journals), fuentes fundamentales para las historias azucareras del Caribe: Le Journal des Fabricants de Sucre, The Sugar Cane y The Louisiana Planter and Sugar Manufacturer", Asclepio, 67 (1): p079. doi: http://dx.doi.org/10.3989/asclepio.2015.05

RESUMEN: Este ensayo destaca la importancia de las publicaciones comerciales de la segunda mitad del siglo XIX y la primera del siglo XX (trade journals) dedicadas al azúcar de caña como el estadounidense Louisiana Planter and Sugar Manufacturer, el francés Le Journal des Fabricants de Sucre, y el británico The Sugar Cane. Su abundante y confiable cobertura sobre la producción, el procesamiento y el mercadeo de este producto básico o mercancía tropical, es decir, la cadena de mercancías, las convierten en fuentes indispensables para el estudio del azúcar en el Caribe. El trabajo se centra en un estudio de caso del Louisiana Planter and Sugar Manufacturer al explicar su gestación e internacionalización, clientela, y analizar su cobertura del Caribe, en particular Cuba, Puerto Rico y la República Dominicana, con referencias al Caribe danés, francés, holandés e inglés.

PALABRAS CLAVE: Historia del Caribe; Azúcar; Historia azucarera; Azúcar de caña; Revistas azucareras; Cadena de mercancías; Publicaciones comerciales; Factoría central; Central azucarera; Louisiana Planter and Sugar Manufacturer; The Sugar cane; Le Journal des Fabricants de Sucre.

TRADE JOURNALS AS FUNDAMENTAL SOURCES FOR CARIBBEAN SUGAR HISTORIES: LE JOURNAL DES FABRICANTS DE SUCRE, THE SUGAR CANE AND THE LOUISIANA PLANTER AND SUGAR MANUFACTURER

ABSTRACT: This articule highlights the importance of sugar trade journals from the second half of the 19th century and the first of the 20th century, such as the US-Louisiana Planter and Sugar Manufacturer, British-The Sugar Cane, and French-Le Journal des Fabricants de Sucre. Their abundant and trustworty coverage of Caribbean sugar production, processing and marketing (namely, the commodity chain) makes them an indispensable source for Caribbean sugar histories. The paper centres on the Louisiana Planter and Sugar Manufacturer as a case study by explaining its establishment and international expansion, clientele, and its coverage of the Caribbean, particularly Cuba, Dominican Republic and Puerto Rico but also with references to the British, Danish, Dutch and French Caribbean.

KEY WORDS: Caribbean history; Sugar; Sugar cane; Trade journals; Sugar journals; Commodity chain; Commercial publications; Central factory; Sugar central; Louisiana Planter and Sugar Manufacturer; The Sugar Cane; Le Journal des Fabricants de Sucre.

Copyright: () 2015 CSIC. Este es un artículo de acceso abierto distribuido bajo los términos de la licencia Creative Commons Attribution-Non Commercial (by-nc) Spain 3.0. 
Una de las fuentes más importantes para la investigación histórica de la agroindustria de la caña de azúcar en el Caribe la constituyen las revistas comerciales (trade journals), que se originaran en las metrópolis imperiales durante la segunda mitad del siglo XIX y continuaron existiendo hasta el siglo XX. Sin embargo, la historiografía azucarera caribeña, con excepción de Beachey (1957), Iglesias García (1998), McAvoy (2003) y García Muñiz (2010), no se ha referido a ellas con el rigor y frecuencia que merecen, aún cuando es probable que el azúcar haya sido una de las primeras mercancías o de los productos básicos tropicales (http:// www.commodityhistories.org) con esta clase de publicación. Dicha situación sorprende porque estas revistas comerciales sobre el complicado y competitivo mundo de la producción y comercio de los azúcares y las mieles, ofrecen información valiosa, la mayoría de las veces confiable, para los involucrados en este negocio de carácter mundial.

Por lo regular, estas revistas se centraron en los aspectos económicos, ambientales, comerciales, tecnológicos, técnicos y científicos de la producción de azúcar de caña, en particular los aspectos de su cultivo y transportación a la fábrica central (usine, en francés), manufactura de mieles y azúcar centrifugada o cruda (96o), refinación, y venta de refino (en general, los dos primeros procesos, en el Caribe, y los dos últimos, en la metrópolis). Los autores de los artículos eran, en su gran mayoría, especialistas del tema en cuestión. Su clientela fue también cualificada (propietarios de plantaciones, colonos, fabricantes de maquinaria agrícola y manufacturera, químicos, ingenieros, administradores de centrales y refinerías, corredores de azúcar, banqueros, financieros, casas de seguros, entre otros), probablemente vinculada o relacionada a la producción, la transportación o la venta del dulce, y muchas veces en competencia entre sí. Con cierta regularidad, estos destinatarios se tornaban en corresponsales, al enviar cartas y notas informativas y aclaratorias desde sus centros de trabajo en el Caribe o en la misma metrópoli. No fueron revistas de circulación popular por la especialidad de su tema y porque se subvencionaron por suscripción y enviaron por correo. Es probable que la fuente de financiación más importante fuera los anuncios de fabricantes de maquinaria e implementos para el cultivo de caña y su manufactura en dulce, de casas de corredores de azúcar, ingenieros consultores y otros muchos de esa índole.

Este ensayo es un estudio de caso de la revista azucarera Louisiana Planter and Sugar Manufacturer (LPSM), cuya publicación se extendió de 1888 a 1929.
En este analizamos los orígenes del LPSM, su cuerpo editorial, su expansión e internacionalización, y las diferentes secciones principales del órgano, con énfasis en su cobertura de las Antillas hispanas: Cuba, Puerto Rico y la República Dominicana. También hacemos breves referencias a los territorios franceses, ingleses, holandeses y daneses, así como a otros aspectos fundamentales de la producción, procesamiento y mercadeo del azúcar de caña. A manera de trasfondo, se discuten, hasta temprano en los 1900, las otras dos revistas azucareras comerciales previas más importantes: Le Journal des Fabricants du Sucre: organe politique de la sucrerie indigéne et coloniale (LJFS), publicada en París, Francia, de 1864 a 1948, y The Sugar Cane (TSC), publicada en Manchester, Gran Bretaña, de 1869 a $1898^{1}$.

El valor de estas revistas radicó en la amplitud y profundidad de su cobertura geográfica y temática. En el caso de las revistas británica y francesa se publicaron artículos y noticias (aunque no exclusivamente) sobre la agroindustria cañera de sus colonias tropicales o, y en el caso del LPSM, de países en la esfera de influencia del imperio estadounidense, como Cuba y la República Dominicana. La revista francesa, el LJFS, cubrió principalmente su agroindustria remolachera indígena y de otros países europeos de clima templado. Como se puede ver en la ilustración 1 , la relación entre los productores metropolitanos y coloniales en el mercado interno francés no era amistosa. El azúcar de caña dominó el mercado mundial durante la primera parte del siglo XIX, pero la de remolacha aumentó de 14.3 por ciento en 1850 a un 69 por ciento de la producción mundial en 1889. En 1913, como consecuencia de la eliminación de subsidios a la producción y exportación del azúcar de remolacha en Europa, estaban más o menos parejas (Crespo, 2006).

En los EEUU, el azúcar de remolacha tuvo su propulsor mayor en la persona del Dr. Lewis S. Ware, quien por 30 años editó, de su peculio, la revista The Sugar Beet. Otra contribución suya importante fue la acumulación de una colección de 12,000 volúmenes sobre azúcar de caña y remolacha, la cual donó al Franklin Institute, de Filadelfia (Palmer, 1918), que, a su vez, la enriqueció por un sinnúmero de años. En ella, se encuentran colecciones casi completas del LPSM, LJFS y TSC, acompañadas de otros trade journals azucareros y una gran cantidad de libros especializados en estos productos básicos agrícolas ${ }^{2}$. La colección completa del LPSM (Vol. 1, 1888-Vol. 83, 1929) está disponible en papel en la National Agricultural Library en Washington, D.C., y en línea en el Hathi Trust Digital Library 


\section{Ilustración 1}

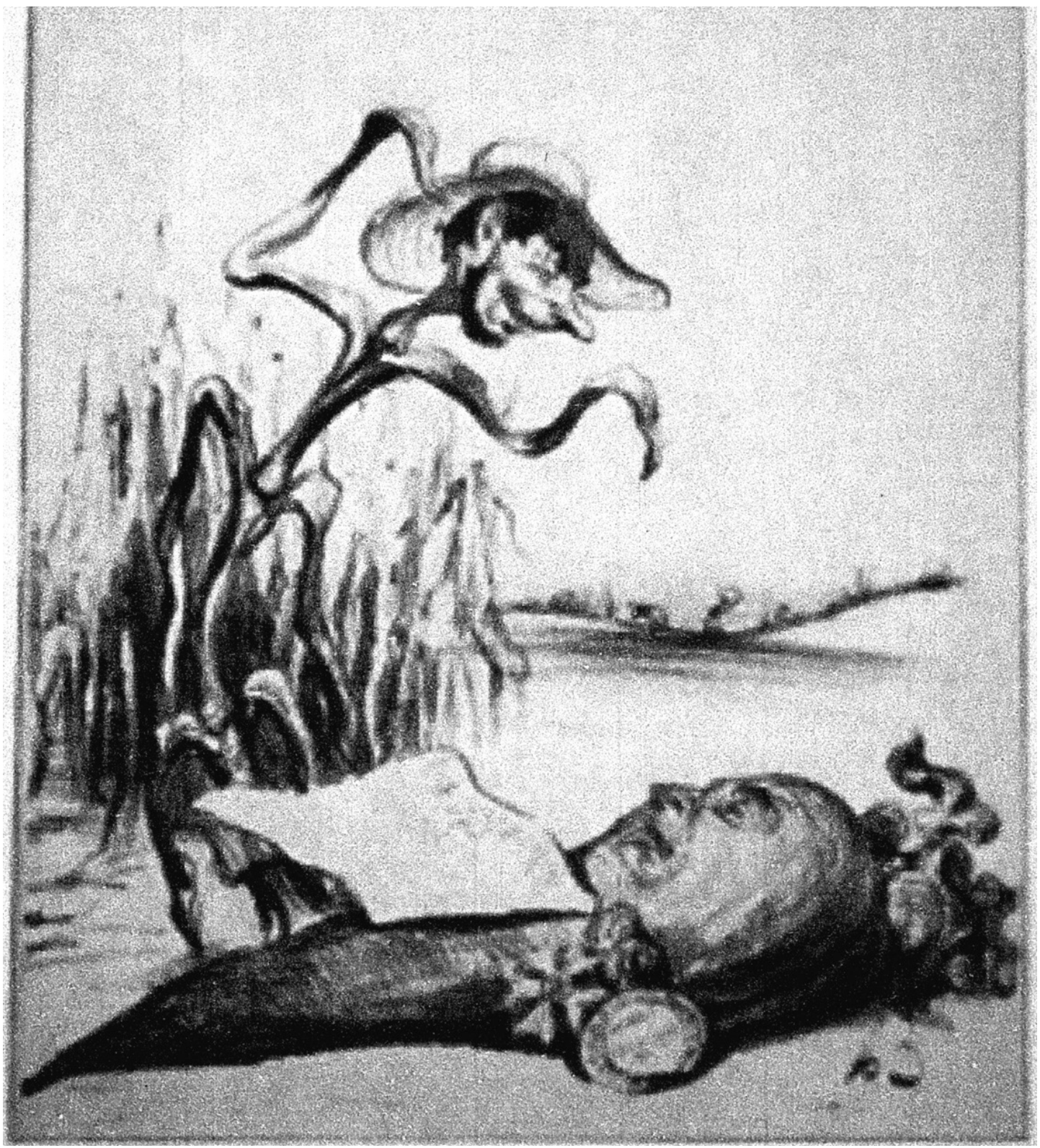

(http://catalog.hathitrust.org/Record/000674886), del Vol. 28 (1902) al Vol. 63 (1919), con búsquedas limitadas del Vol. 70 (1923) al Vol. 83 (1929). Por su parte, la colección completa del TSC (Vol. 1, 1869-Vol. $30,1899)$ se encuentra disponible en papel también en la National Agricultural Library, y del 1874 al 1898 en la New York Public Library, con consulta limitada en la Hathi Trust Digital Library (http://babel.hathitrust. org/cgi/pt?id=nyp.33433008135281). El LJFS se encuentra en la Biblioteca Nacional de Francia de 1874 al 1948, y en Wayne State University del Vol. 1 (1861) al Vol. 3 (1863), y del Vol. 12 (1891) al vol. 54 (1913).
Cabe recordar las revistas publicadas en países tropicales, cultivadores de caña y productores de azúcar, cuya excelente calidad son un reflejo del grado de desarrollo de la agroindustria y el conocimiento tecnológico de la sacarocracia local en cierta coyuntura. Tres de ellas merecen ser consultadas: la Revista de Agricultura, del Círculo de Hacendados de Cuba, Timehri, the Journal of the Royal Agricultural and Commercial Society of British Guiana (https://archive.org/details/timehrijournalr06guiagoog), y el Hawaian Planters' Monthly ${ }^{3}$. En cada una se incluyen referencias, incluso testimonios, de experiencias en los otros territorios productores. 


\section{LE JOURNAL DES FABRICANTS DU SUCRE - UNA REVISTA AZUCARERA FRANCESA}

El LJFS se fundó en 1860 por Jean-Baptiste Dureau, quien fue gerente de una refinería en Nantes y de centrales azucareras en Ardonne, Arras, Bourdon y Sarlieve, Francia, y plantador en Luisiana, EEUU (Palmer, 1914). Esta no fue la primera publicación francesa del azúcar. Durante un año antes circuló el Bulletin des Sucres françaís et étrangers, de la Agence Agricole. A la muerte del fundador, su hijo Georges se hizo cargo del LFJS hasta que la vendió a La Societé de Publications Industrieles et Agricoles en 1919, aunque continuó como editor. EI LPSM afirmó que "la alta posición lograda por los Dureau con su revista durante los últimos 50 años ha sido prácticamente la base sobre la cual la literatura francesa del azúcar se ha levantado" (“Journal", 1919).

Como adelantamos, el plato fuerte del LJFS fue la agroindustria remolachera europea, pero también cubrió las colonias caribeñas francesas y de otras metrópolis. Desde su primer número, fechado el 12 de abril de 1860, M. P. L. Fernández, un plantador de Cuba, publicó cifras del costo de producción en diferentes haciendas y de las exportaciones azucareras de la isla (“Le Journal", 1865). El reformista agrícola gallegocubano, Ramón de La Sagra, autor de los 13 volúmenes de la Historia física, política y natural de la isla de Cuba (1838-1857), fue otro de sus colaboradores. A principios de 1865, de la Sagra contribuyó desde París, con dos artículos, posiblemente desconocidos hasta ahora, sobre la separación del cultivo de la caña y la fabricación de azúcar (Sagra, 1865a, 1865c). También polemizó por carta con Cristóbal Madan, "uno de los cuatro jinetes del Apocalipsis sacarócrata" (Moreno Fraginals, 1986), sobre su opinión del impacto de la abolición de la esclavitud en la producción azucarera (Sagra, 1865b) ${ }^{4}$. Su última contribución fue sobre la Junta de Información de 1867, encargada de proponer reformas al régimen político, administrativo y económico de Cuba y Puerto Rico, de la cual era miembro designado (Sagra, 1867).

En el entre siglo, varios artículos sobre Puerto Rico, de la pluma del ponceño (hijo de corso) Mathieu Lucchetti, de la Compagnie des Sucreires de Porto Rico, propietaria de la Central Fortuna, ofrecen un análisis hasta el presente desconocido del impacto de los problemas del cambio de moneda, del mercado y de los estragos del huracán de 1899 , en los años de transición como colonia del imperio español al estadounidense, de 1895 a 1905, año en que muere ahogado.

\section{THE SUGAR CANE - UNA REVISTA AZUCARERA BRITÁNICA}

EI TSC comenzó su publicación en la ciudad industrial de Manchester, Gran Bretaña, en 1869, para llenar el vacío en la prensa inglesa (metropolitana y colonial) de "un órgano especial" que sería "el medio de comunicación entre los interesados directamente en el crecimiento de la caña y la manufactura del azúcar en todas las partes del mundo". Sostuvo que los intereses de los plantadores coloniales y los refinadores británicos no eran contradictorios, así que "ambos serán igualmente representados en estas páginas" ("Address", 1869). Ya se hizo claro que, por su dependencia en el producto, incluiría cuestiones de importancia para el Caribe colonial británico, pero también sobre otros intereses azucareros donde estuvieran presentes el capital y la maquinaria británicas.

Su fundador, Alfred Fryer, tuvo conocimiento directo de la producción y refinación del azúcar de caña ya que invirtió en plantaciones en Antigua y por ser socio administrador de la refinería Fryer, Benson \& Foster en Manchester. Hizo nombre como el inventor del Fryer Concretor, un producto que no tuvo aceptación en el mercado, y defendió el libre comercio con la misma furia que atacó los subsidios e impuestos.

La cubierta del TSC contó con el apoyo de corresponsales que informaron desde países con una agroindustria significativa de producción azucarera. De Puerto Rico, colaboraron de los 1870's a los 1900's, entre otros, M. Lee, Santiago [James] McCormick, Roberto Graham, Gustavo Cabrera y Richard H. Burton. Este último, un británico proveniente de St. Vincent, "con una experiencia de 30 años como plantador práctico, en todos sus grados, capataz, gerente, apoderado, propietario", fue el principal corresponsal (Burton, 1874) y hasta ahora sus contribuciones han pasado inadvertidas. Las factorías centrales, que tienen su origen en la agroindustria remolachera europea y que se replican primero en Guadalupe y Martinica, fueron su tema favorito y uno de los más debatidos en la época (Schnakenbourg, 1984). Sobre estas, aportó con dos informes: uno de las usines en Martinica en 1874 (Burton, $1875 a, 1875 b)$, y otro sobre la maquinaria y el funcionamiento de la Central San Vicente, la primera en Puerto Rico, construida en 1873 por Leonardo Igaravídez y equipada con maquinaria francesa Cail (Burton, $1874)^{5}$. Sus escritos, traducidos al portugués por J. José Carneiro da Silva, sirvieron de guía para la construcción de la primera factoría central en Brasil, localizada en el distrito de Quissama, Rio de Janeiro. Carneiro de Silva afirmó: "El origen de la Factoría Central en Brasil 
se deriva de The Sugar Cane"(Carneiro Da Silva, 1877). En Jamaica, Burton no tuvo suerte con el fracaso del proyecto para establecer la compañía "The Jamaica Central Sugar Factories Company (Limited)" ("Central", 1874). Por su importancia en la época, las referencias a las construcciones de factorías centrales en distintos países - prácticamente todas las Antillas, Argentina, Brasil, Ecuador, EEUU (Florida, Luisiana y Texas), Guayana Británica, México, Perú y Surinam - se repiten en el LJFS, TSC y LPSM durante las últimas décadas del siglo XIX y principios del siguiente.

Sobre Cuba, el TSC reprodujo unas cartas privadas de Moriz [Maurice] Weinrich, publicadas primero en el Organ des Central-Vereins für Rubensucker-Industrie, de Austria, probablemente por sus vínculos anteriores con el azúcar de remolacha. En ellas, Weinrich escribió que "no hay duda de que nos vamos rápido a la ruina" por la guerra civil, que lleva 10 años, y por los impuestos exorbitantes a las exportaciones de azúcar (Weinrich, 1881). Muy poco y breve se publicó sobre la República Dominicana, por lo menos hasta la primera década del siglo XX. En 1892, se informó que la sequía había causado una baja en la producción, de 30,000 a 18,000 toneladas de azúcar, de las cuales 12,000 provenían de San Pedro de Macorís, "que se está convirtiendo en un sitio importante aunque 10 años atrás era solo una villa pesquera insignificante" ("Santo Domingo", 1892). No podía faltar el controvertido William L. Bass, quien, ya vendido su Ingenio Consuelo en San Pedro de Macorís, se anunció como un "experto en plantaciones de azúcar de caña", y el TSC lo refrendó al decir que "no había duda que estaba cualificado para dar consejo desde un punto de vista práctico" (TSC, 1902). Pocos años más tarde publicó en los EEUU unas revistas contra el Trust del Azúcar, The Gator y The Sugar Sentinel.

No todas fueron noticias tecnológicas o económicas. A fines de octubre de 1879, el TSC informó que en la colonia danesa de St. Croix, tras la expiración de sus contratos de trabajo, unos trabajadores se levantaron y asesinaron a un plantócrata y dos policías, e incendiaron varias plantaciones y negocios. El desorden se controló con fuerzas de seguridad de St. Thomas y de dos naves, una francesa y la otra británica ("The Outbreak", 1879).

\section{GESTACIÓN DEL LOUISIANA PLANTER AND SUGAR MANUFACTURER}

Al momento del número inicial del LPSM en 1888, el TSC era la única revista en inglés y tuvo una tirada mensual. Según alegó el LPSM, esta publicación estaba "bajo el patronazgo de algunos manufactureros de maquinaria" y se dedicaba "solamente al lado mecánico y muy poco al agrícola" ("Twenty", 1913). El LPSM tuvo como patrocinador al Louisiana Sugar Planters' Association (LPSA), también la organización fundadora breves años antes de la Escuela Azucarera Audubon y una estación experimental (Heitmann, 1987). Comenzó y se mantuvo como un semanario "técnico, de corte educativo con el propósito de sostener y promover la industria azucarera de la caña en el mundo, que lleva una década en decadencia ante la competencia avasalladora del azúcar de la remolacha" ("Twenty", 1913).

La LPSA ubicó el LPSM bajo el control editorial, gerencial y corporativo de John Dymond (1836-1922). Al igual que los fundadores de LJFS y TSC, este tenía experiencia práctica en la industria cañera ("John Dymond", 1922). Canadiense de nacimiento, Dymond se crió en Ohio y más tarde se radicó en Nueva York. Allí estableció la sociedad Dymond \& Lally, que llevó a cabo múltiples negocios en azúcar y café en esta ciudad y Nueva Orleans. En 1868, tras la Guerra Civil, la sociedad adquirió las plantaciones azucareras de Belair y Fairview en Luisiana; Dymond se mudó allá para atenderlas. Ese fue el comienzo de su legendaria vida como plantador. Fue el primero en varios aspectos de la industria en el estado como: la instalación del tranvía ligero para transportar caña, el pesaje de la caña al llegar a los molinos (lo que permitió establecer una base de determinación de costo), la introducción del polariscopio en la fábrica, la instalación del primer molino de seis rollos, y la instalación de un condensador seco al vacío. Fue, además, el responsable de obtener los servicios expertos del agrónomo Dr. W. C. Stubbs para la estación experimental de Audubon Park. Incursionó en la política por el Partido Demócrata. Favoreció la supremacía blanca, la autonomía del estado y el proteccionismo en cuanto al comercio internacional. Incluso, se le nominó, aunque declinó, la candidatura para la gobernación.

A la larga Dymond no tuvo mucho éxito en la política, pero sí aprovechó sus lazos con los plantadores de Luisiana y con los pares de la agroindustria en otros paralelos para obtener información de primera mano. Así identificó y obtuvo colaboraciones regulares de corresponsales, muchas de las cuales eran de los "trotamundos del azúcar" (sugar tramps) de Luisiana (García Muñiz, 2010), y otras de los más prominentes conocedores y científicos de la época, algunos de los cuales mantuvieron columnas regulares por muchos años. Entre ellos, 
se destacaron Georges Dureau, editor del LJFS; Robert Hennig, editor del Deutsche Zuckerindustrie de Berlín, Alemania; P. Boname, de la Estación Experimental de Mauricio, y Tomás Delorme y Allain, del Diario de la Marina, La Habana, Cuba. También contó con los Sres. Willet y Gray, de la revista neoyorquina de estadísticas azucareras Willet \& Gray, e informes semanales de los corredores de azúcar, M.G. Wanzor \& Co. (más tarde de Lamborn \& Co., Inc.), así como varios colaboradores del New York Journal of Commerce. Truman G. Palmer, experto en la industria azucarera de remolacha, cubrió las incidencias legislativas y ejecutivas en Washington, D. C., y de producción y mercadeo en Europa. Otro corresponsal, S. G. Ruegg, que escribía desde Europa, publicó, entre otras, una serie informativa sobre manufactureros británicos de maquinarias como David Cook \& Son, Mirrlees Watson \& Company, de Glasgow, y Fawcett, Preston \& Co. Ltd., de Liverpool (Ruegg, 1922a, 1922b, 1922c). Desde Queensland, Australia, colaboró A. E. Lavis, vinculado a la estación experimental donde trabajaba el respetado Dr. Walter Maxwell. C. G. Heisier, de Honolulu, fue el corresponsal de las Islas Hawái. El Dr. H. Prinsen Geerligs, una reconocida autoridad holandesa, inicialmente químico azucarero que hizo su fama en la agroindustria azucarera en Java, colaboró sobre Europa y con ensayos cortos científicos e históricos, como el informe sometido a la Liga de las Naciones en 1929 (Prinsen Geerligs, 1929). El Dr. Harvey D. Wiley, principal promotor gubernamental de la fallida autosuficiencia sacarina de los EEUU en el siglo XIX, mejor conocido por el Pure Food and Drug Act de 1906, fue un corresponsal irregular, como muchos otros expertos azucareros, químicos e ingenieros azucareros.

Las suscripciones al LPSM Ilegaron de Europa, Australia, Egipto, India, Sudáfrica, Mauricio, Java; las Filipinas, las colonias caribeñas británicas, danesas, españolas, francesas y holandesas; México; la Guayana Británica; Surinam; Argentina; Brasil, y las islas Hawái en el Pacifico. El semanario llegó a tener una circulación en unos 40 países. Una muestra de su éxito fueron los dos intentos de replicarlo, uno en Queensland, Australia, y el otro, en México, ambos fundados por dos corresponsales suyos ("Our Anniversary", 1904). El primero, The Sugar Journal and Tropical Cultivator, tuvo una tirada mensual y parece ser que duró de 1892 a 1905. Del otro, el Mexican Sugar Planter, no hemos logrado identificar las fechas ni ubicar siquiera un número.

\section{INTERNACIONALIZACIÓN: CUBA, MÉXICO, NUEVA YORK, BRASIL Y LA RADIO}

El 1ro de febrero de 1913, el LPSM inauguró una sucursal en La Habana. Irene A. Wright, quien ya llevaba un año con variadas contribuciones publicadas, fue nombrada a cargo de la oficina. Nacida en el estado remolachero de Colorado, EEUU, Wright se graduó de Stanford University, California, y estaba radicada en la ciudad, donde colaboraba con el Diario de la Marina y el Havana Post. Por su cuenta, publicó una revista mensual agrícola, The Cuba Magazine ("Our Havana Office", 1913). En agosto de 1913, durante la celebración de su $25^{\circ}$ aniversario y a cinco meses de abrir la sucursal de La Habana, el LPSM sorprendió con la publicación de El Mundo Azucarero, una revista mensual en español "de la cubierta del frente hasta la de atrás" ("Aviso", 1913) ${ }^{6}$. De hecho, fue la primera revista comercial en castellano dedicada a un producto agrícola tropical (Brand, 1948).

En febrero de 1914, Wright cesó en sus funciones y fue reemplazada por A. Hall, un "hombre del azúcar", graduado de la escuela de Audubon, con tres años en la estación experimental de Tucumán, Argentina, y un buen dominio del español ("Our Havana Office", 1914a). Tres años más tarde, H. A. Granary lo sustituyó. En diciembre de 1920, este último renunció a su vez para comenzar como asistente del gerente general del Central Hershey, construida por los intereses chocolateros de Milton Hershey, de Pensilvania, EEUU. R. G. Tillery, un técnico azucarero con muchos años de experiencia con la Cuban American Sugar Co., comenzó como nuevo gerente ("A Change", 1920). En julio de 1924, se anunció que el ingeniero azucarero, Wiley D. Stephenson, con 18 años en la industria azucarera de Luisiana y siete en Cuba, se encargaría de la sucursal de La Habana ("Our Havana Office", 1914b).

En julio de 1914, el LPSM abrió una oficina en Ciudad México. Se esperaban "vastos negocios" pues "en los pasados dos años no se hicieron reparaciones ni mejoras en las fábricas porque las condiciones amenazadoras lo hacían imposible". Ahora, con "la llegada de la paz", comenzaría "un renacer tremendo de la actividad manufacturera azucarera, en especial por los altos precios del azúcar causados por la guerra europea" ("Office", 1914). En 1919, el LPSM se expandió a la ciudad de Nueva York, con el nombramiento de John S. Dennee como representante de sus dos publicaciones: el LPSM y El Mundo Azucarero. Nativo de Nueva Orleans y graduado de Tulane University, Dennee fue empleado del Southern RaiIways en sus oficinas de Boston y Nueva York. Tam- 
bién laboró en Hawái, México y las Filipinas (“John S. Dennee", 1919). Al año, el mayor G. C. Comstock, también de Luisiana, recién llegado de la guerra, fue nombrado jefe de la oficina en Nueva York. Parece ser que no tenía mucha experiencia porque se esperaba que "se hiciera rápido un amplio círculo de amigos y conocidos entre los hombres del azúcar en Nueva York, que se han convertido en numerosos, competentes y progresistas agentes de la industria azucarera en el mundo" ("Major", 1920).

A la muerte de Dymond en 1922, su hija Florence se hizo cargo del LPSM y se anunció la adición al cuerpo editorial de los doctores F. W. Zerban y W. R. Dodson (Dr. F. W. Zerban, 1922; Dr. W. R. Dodson, 1922). El primero, graduado de la Universidad de Munich, sucedió al Dr. C. A. Browne como químico de investigaciones en la Estación Experimental de Audubon Park. También estuvo vinculado a otras estaciones experimentales en Lima, Perú, Tucumán, Argentina, y la Estación Experimental de la Asociación de Productores de Azúcar de Puerto Rico. El Dr. Dodson, doctorado en química de Harvard University, fue profesor en Louisiana State University (LSU) y botánico en la Estación Experimental de Luisiana, además de ser decano de la Escuela de Agricultura de LSU.

A mediados de 1922, se nombró al Dr. R. E. Blouin como editor en jefe. Nacido en Baton Rouge, se graduó de LSU con una licenciatura en ciencias en 1891 y una maestría en ciencias en 1892. Más tarde, hizo estudios graduados en la University of Chicago en 1896 y 1897. Su experiencia de trabajo en investigación era "impresionante": fue químico asistente (1891) y químico (1898) en la Estación Experimental Estatal de Luisiana, y director de la Estación Experimental de los Plantadores de Azúcar de Hawái (1900) (“Our Editorin Chief", 1922).

El Dr. Blouin continuó con la política de expansión del LPSM hacia Latinoamérica. En 1924, estableció una sucursal en la Rua da Quitanda, Río de Janeiro, Brasil, a cargo de su representante, el Dr. Joao Brazil Silvado. La justificación fue "el rápido desarrollo e importancia de la industria azucarera de Brasil nos ha llevado a tener un representante directo en este país" ("Our Brazilian Office", 1921). Pero fue más allá de establecerse físicamente para cubrir las noticias de los principales países productores hemisféricos. El 22 de marzo de 1924, a petición de productores de azúcar en Suramérica y el Caribe, comenzó la transmisión diaria de informes sobre el mercado azucarero por la estación radial Westinghouse KDKA, de Pittsburgh, en el horario de 7:40 p.m. a 8:00 p.m. ("Sugar", 1924).

\section{COBERTURA DEL CARIBE}

En sus 41 años de existencia, la fortaleza del LPSM, en comparación con las otras revistas azucareras, fue su cobertura de los más variados aspectos de la agroindustria cañera. Se puede afirmar que cubría todas las etapas de la cadena de mercancías o productos básicos (commodity chain) tropicales al incluir noticias sobre la producción, el procesamiento y el mercadeo del azúcar (sin llegar al consumo) (Talbot, 2002). La producción en el trópico era su fortaleza, especialmente los territorios que eran su lema desde el 1920: "Esta publicación circula dondequiera que se produzca azúcar pero principalmente en Cuba, Porto Rico (sic), Luisiana, México y Hawái" (LPSM, 1920). Sin embargo, las secciones de esos países no agotaron su cubierta porque incluía noticias sobre ellos en otras partes del semanario. Hay temas relacionados a la compra y venta del dulce, como los asuntos controversiales de las tarifas y los precios de venta a los refinadores, que son recurrentes. Muy notable fue que desde temprano, a cinco años de su descubrimiento en 1879, comenzó a seguirle la pista a sustitutos del azúcar como la "sacarina" y al "valzin", también llamado "dulcin" en honor a la Dulcinea de Don Quijote ("A New Substitute..." 1893; "Former...", 2003).

Los lazos personales y profesionales de Dymond (y sus sucesores en el cuerpo editorial) con el personal técnico, administrativo y gerencial de centros azucareros en todo el mundo le dieron una ventaja sobre cualquier otra publicación similar al obtener acceso continuo a información privilegiada. La enorme mayoría de esos lazos provenían de la práctica de este personal azucarero en la agroindustria del dulce de Luisiana, o por sus estudios en LSU. Un ejemplo temprano fue una extensa carta de Frank Coombs, químico residente en el Ingenio Elizalde, en Isabel, Cuba, fechada el 17 de marzo de 1893, en la cual se refería a las discusiones sobre el uso de sacos o toneles en el empaque de azúcar cruda; las ventajas en tamaño, cantidad y calidad del guarapo de la variedad Cristalina sobre la Red Cavengerie, y las diferencias del proceso de defecación con cal, incluyendo referencias comparativas a sus experimentos pocos años antes en la Central Calumet, de Luisiana (Coombs, 1893). En otra misiva, informó que en Matanzas visitó la refinería de mieles de los Sres. Bea, Bellido \& Co., que con maquinaria de Weston y Westinghouse, se prestaba a comenzar operaciones ("Private Advice's", 1893).

Esa singular conexión humana se manifestó en las menciones directas de los "trotamundos del azúcar" en el LPSM a su regreso estacional al lar nativo. Breves 
oraciones al pie de la página del semanario se dedicaron a los trabajadores especializados como L. Litty, encargado de calderas "con muchos años de experiencia", quien "retornó a su casa en Nueva Orleans... de la Usine St. Madelaine, Trinidad, [y Tobago] donde estuvo empleado en la pasada zafra" ("Personal", 1912). Un examen del número de la primera semana de enero de 1905 revela más de 50 solicitudes de empleo, entre ellas, un químico jefe y también un azucarero disponibles para Puerto Rico, Cuba o México, o un tal F. D. Millet, de Lucy, Luisiana, como herrero y ruedero ("Situations Wanted", 1905). A estos "trotamundos del azúcar" aplica el dicho que "no paraban la pata", pues muchos se trasladaron de zafra en zafra al continuar de Luisiana a la del Caribe, México, Centro o Sur América, y a veces a la de remolacha en EEUU, ya que una seguía a la otra en el calendario agrícola.

Los hermanos Boyd, hijos del presidente de LSU, T. D. Boyd, son muestras ejemplares de estos "trotamundos del azúcar". T. D. Boyd, hijo, fue nombrado a cargo de las centrales en manos de la Cuban Sugar Plantations Inc., del National City Bank, en Cuba, como consecuencia de la debacle de precios de los 1920 ("Thos. D Boyd, Jr.", 1922). Antes trabajó en Calumet y Shadyside (Luisiana), Central Tinguaro (Cuba), Ingenio San Antonio (Nicaragua), Central Constancia y United Sugar Companies (México), y la South Porto Rico Sugar Company (Puerto Rico y República Dominicana). Su hermano, Overton, laboró en la industria remolachera de California; la azucarera de Cuba, México, Haití, y República Dominicana, de donde describió a su padre un dramático asesinato de un ingeniero civil de Luisiana por los gavilleros (García Muñiz, 2010). Su último trabajo fue en la esfera de influencia azucarera británica al ser nombrado como tecnólogo azucarero a cargo de la estación experimental del Imperial College of Agriculture en Trinidad y Tobago ("Overton", 1957), un reconocimiento al valor de las investigaciones realizadas en Luisiana. Desde allí publicó en el LPSM, a raíz de la muerte de Sir Francis Watts, un ensayo sobre la enorme contribución de este "científico pionero... quien sin duda ha hecho más que ninguno de sus colegas en mejorar las condiciones agrícolas y de cultivo en las Indias Occidentales" británicas (Boyd, 1924).

En el caso de Cuba, la primera sección se tituló "La Habana", firmada por "T. D.," (probablemente Tomás Delorme y Allain), pero desde el 1913 apareció el "News Letter From Our Havana Office", con una cantidad y calidad de información, inclusive de rumores, impresionantes. Cada entrega semanal de Irene Wright contenía variadas informaciones según el periodo del año azucarero: desmontes de tierras, aperturas o cierres de centrales; compras, reparaciones y adiciones de maquinaria; visitas de técnicos y personalidades; nuevas experimentos de equipos de labranza, cultivo y de manufactura; asuntos ferroviarios; migraciones de boricuas, jamaiquinos y haitianos; paros de estibadores; ventas y precios de azúcares; nuevos libros publicados; noticias del clima, en otros muchos temas.

Además, el LPSM incluía artículos individuales valiosos como, por ejemplo, el olvidado informe del multifacético Nöel Deerr a la Secretaría de Agricultura, Comercio y Trabajo, titulado "The Cane Sugar Industry" (1914a, 1914b), reproducido en El Mundo Azucarero y republicado por Zanetti, Venegas Delgado y García Muñiz (2010). En diciembre de ese año, el LPSM se preguntaba la posibilidad de la expansión de la caña en las sabanas de la parte oriental, tal como había acontecido en las Guayanas ("Cuba's", 1914). Sobre las fundiciones cubanas de maquinaria, se publicó un artículo descriptivo de Manuel Galdo \& Co., empresa radicada en la ciudad de Cárdenas, en la provincia de Matanzas, donde "la manufactura de maquinaria azucarera, junto con el diseño, equipamiento y levantamiento completo de la fábrica azucarera era su principal especialidad" ("The Shops", 1917). En septiembre de 1920, en plena "Danza de los Millones", Granary escribió un informe sobre las nuevas construcciones de centrales y refinerías, las reorganizaciones de las compañías azucareras, y los cambios de propietarios "como nunca antes se había hecho en la historia de Cuba" (Granary, 1920). Hubert Edson, un conocedor como pocos de la industria de Luisiana y el Caribe, publicó en 1928 un breve ensayo sobre las ventajas de la refinación del azúcar en Cuba y no en los EEUU (Edson, 1928).

La importancia de la mecánica (extracción del guarapo de la caña que se obtiene por la trituración de los molinos) y de la química (calidad de la sucrosa obtenida del guarapo de la caña) en el proceso de elaboración de azúcar son temas muy discutidos en el LPSM. Estas siempre fueron acompañadas con referencias históricas y a las últimas investigaciones y experimentos en variadas centrales de distintos países azucareros. En 1923, el reputado químico Guilford L. Spencer, de la Cuban American Sugar Co., reconoció que el gran avance tecnológico fue el desarrollo de los molinos, pero argumentó que "la moderna factoría de caña está controlada químicamente en cada etapa de la manufactura" y se requiere que los superintendentes y sus asistentes "tengan buen entrenamiento químico” (Spencer, 1923). 
La cobertura de Puerto Rico fue fácil para el LPSM. La isla era más pequeña que Cuba, y tenía mayor presencia y peso de los "trotamundos del azúcar"; además, el control imperial directo de los EEUU le favoreció. Su cubierta de la Guanica Centrale, siempre la más grande de la isla y la más grande del mundo en un momento, fue excepcional al publicar noticias e informes detallados de su maquinaria y funcionamiento, como los de D. L. Thomson (1904) y John Bovell (1911), este último en el West Indian Bulletin, órgano del Imperial College of Tropical Agriculture. Además, las fotografías del central y su maquinaria fueron excelentes, como usualmente eran las publicadas en todos sus números.

La sección de "Letter from Puerto Rico" abarcó los temas principales y su seguimiento a lo largo de los años, lo que permite estudiar la expansión del capital estadounidense por medio de la compra de centrales boricuas en la agroindustria. Sobresalen varios trabajos individuales, como el de George W. Rolfe, de MIT, sobre la manufactura del azúcar, y otro del agrónomo J. T. Crawley sobre la cuestión agrícola (Rolfe, 1912; Crawley, 1913). Su cubierta de las centrales puertorriqueñas fue frecuente y pormenorizada. Por ejemplo, en 1926, el LPSM revelaba que el Central Mercedita, de la ponceña familia Serrallés, refinó un azúcar granulada por el proceso "Suchar", que se llamó "Snow White" ("Standard", 1926).

A pesar de los intentos de William L. Bass, la República Dominicana, a diferencia de Cuba y Puerto Rico, no tuvo acceso preferencial al mercado estadounidense hasta los 1960 (Bass, 1902). Con todo y eso, la mayoría de su industria estuvo en manos estadounidenses desde fines del siglo XIX, pero con una producción mucho menor que las otras Antillas hispanas hasta casi la década de los 1940's. El personal azucarero de Luisiana, aunque presente, no fue sustancial. Las noticias a fines del siglo XIX son esporádicas, aumentan un poco en las primeras dos décadas del siglo XX y en los 1920 ya son frecuentes, aunque cubren básicamente el tonelaje producido. La mayoría informaban de la provincia de San Pedro de Macorís, aunque la Central Romana, en la provincia colindante de La Romana, era su favorita. En 1900, H. Garnett, tachero oriundo de la Guayana Británica, informó que "no hay control científico en la factoría, pero la maquinaria, casi toda americana, es muy buena" y agregó que cada vapor de Puerto Rico llega cargado de inmigrantes prestos a trabajar en la caña porque un huracán "destrozó los cultivos" en esa isla (Garnett, 1900). Doce años más tarde, “Dominican"
(1913) afirmó que la inestabilidad política no afecta la producción azucarera porque "75 o 85 por ciento de los trabajadores son importados de las diferentes islas que rodean el país". Se mencionaron, a modo de ejemplo, dos casos de compra de maquinaria azucarera estadounidense: Hugh Kelly \& Co., Central Porvenir, un cuádruple efecto Lillie y F. A. Vicini, General Industrial Co., of Santo Domingo, un horno Fisher de quemar bagazo ("Big", 1912; "Fisher", 1912).

A principios del siglo $\mathrm{XX}$, con capital estadounidense, volvió a renacer la industria azucarera en Haití. En febrero de 1918, A. J. Greif, presidente de la Haitian American Sugar Co., "un viejo y querido hombre azucarero de Luisiana", estuvo pendiente al embarque de una factoría de azúcar a levantarse en Haití ("Louisiana", 1918). Esta empresa contrató a cuatro mujeres químicas de Luisiana para hacerse cargo del laboratorio, la segunda vez que esto sucedió en el mundo predominantemente masculino azucarero ya que la primera fue la Guanica Centrale en 1911 cuando Greif era administrador de esa factoría ("Louisiana", 1920). En un editorial, el LPSM acogió con favor la incorporación de esta empresa en Nueva York porque "desde la Revolución Francesa hace 120 años, la tierra ha permanecido sin cultivar y tiene un valor bajo en el mercado" ("The Redivivus", 1916).

Por su parte, el Caribe colonial británico tuvo buena acogida en las páginas del LPSM. Las secciones de "Barbados" y "British Guiana" predominaron, la primera por ser la primera sugar island inglesa y por la gran aportación de John R. Bovell a las variedades de caña. No sorprende, entonces, que en 1919 el Dr. C. A. Browne, el padre de la historia de la química en los EEUU, redactó expresamente para el LPSM un extenso artículo de su visita a Bovell, entonces director de Agricultura de Barbados (Browne, 1919). Ya antes, en 1897, el LPSM publicó una narración de un barbadense, probablemente blanco, de "un día en una plantación de azúcar en Barbados" (A Barbadian, 1897).

Sobre la Guayana Británica, Wieting \& Richter, Ltd., una compañía de importación y exportación, establecida en la segunda mitad del siglo XIX, todavía hoy en operación en la capital Georgetown, publicó por muchos años un informe de las zafras que incluía "the market", "sugar making", "weather and cultivation", y "labor". En este último apartado, a fines de 1904, comentó que, aún con sequía, había una demanda para trabajadores en el Esequibo y agregó que "el primer barco de culíes de la temporada, el Lena, trajo 500 inmigrantes, y el Clyde 325 inmi- 
grantes, y que el Moy probablemente va a traer 500 inmigrantes, para un total de 1,334, un número que se queda corto de lo solicitado" (Wieting \& Richter, 1904). Al fallecimiento de Carl Wieting, el LPSM afirmó que, con información suministrada por John Dymond de la industria arrocera luisianense, este puede considerarse como el fundador de la próspera producción arrocera de los indo-orientales, a la que se dedicaban al terminar sus contratos en la caña de azúcar (“Carl Wieting", 1919).

Trinidad y Tobago ocupa un tercer lugar y la cobertura fue de la primera isla ya que en la segunda, las plantaciones desaparecieron como resultado, entre otros factores, de la abolición de la esclavitud. El "trotamundos del azúcar", Frank Coombs, que publicó sobre Cuba en 1893, reapareció en 1898 con una extensa descripción de la agroindustria trinitaria que incluyó los aspectos agrícolas, laborales, propiedad y administración, hornos y calderas, molinos, clarificación, y tachos al vacío En ella estableció comparaciones con las agroindustrias de Cuba y Luisiana y la acompañó con impactantes fotografías de las casas de vivienda del plantador y de los "culíes", los tipos de trabajadores "culíes", y el pesaje de una carreta de caña. Al igual que en la Guayana Británica, "el asiático importado - el culí indo-oriental- es el soporte, en ambos, el campo y la factoría:

Prácticamente todo el trabajo manual bruto es realizado por culíes, que están dentro de la factoría, en las clarificadoras, los filtros, las centrífugas y los tanques de mieles. Las mujeres culíes, al igual que los niños mayores, también trabajan en el campo y la factoría (Coombs, 1898).

El LPSM se mantuvo al tanto de otras publicaciones de dentro y fuera del Caribe, como, por ejemplo, el Havana Weekly Report, Financial News (Londres), New Orleans Weekly Picayune, Kew Bulletin (Gran Bretaña), y Revista Azucarera de Cuba, de los cuales reproducía artículos. En 1919, reprodujo un informe publicado en el West India Committee Circular, que trataba sobre las condiciones de trabajo de los inmigrantes de las Islas de Sotavento a la República Dominicana, con la conclusión de que la mayoría se radicó en San Pedro de Macorís y que "el trabajador se siente como en su casa en las plantaciones" ("Labor", 1919). De igual forma, las publicaciones del Caribe conocían del LPSM. A fines de 1913, el LPSM informaba que la revista La Lucha, de La Habana, reprodujo el 17 de noviembre su editorial "El azúcar blanca en Louisiana" que apareció en El Mundo Azucarero.
Finalmente, una investigación con el LPSM, como fuente tiene que examinar la sección "New York". En ella, se incluían variadas noticias sobre distintos aspectos del negocio azucarero que se llevaban a cabo en la urbe, donde se encontraban las oficinas centrales de muchas empresas ubicadas en el Caribe, de los corredores y compradores de azúcar, y manufactureros de equipos, y de las refinerías importadoras de los azucares de la región, en especial la American Sugar Refining Company, conocida como el Trust del Azúcar, en Brooklyn ("New”, 1910). Hoy día, la programada demolición de esta "famosa e infame" refinería para la construcción de complejos de apartamentos de lujo ha causado furor porque es necesario "proteger las estructuras históricas que identifican lo que somos", entre los que "tienen" y los "que no tienen" (Sawa, 2014).

Además, estaban los bancos que financiaron las empresas, agencias de seguros, líneas marítimas, laboratorios y un sinfín de otras actividades relacionadas con esta mercancía tropical. Las visitas de los propietarios de empresas (estadounidenses y caribeños) y los asuntos a tratar se mencionaron con frecuencia. Todavía se carece de una investigación histórica de la relación de la ciudad de Nueva York con la gama de actividades de la agroindustria azucarera del Caribe. Una sección de esa historia debe estudiar el papel del New York Sugar Trade Laboratory, fundado en 1908 a instancias de John Arbuckle, en un momento competidor de Henry O. Havemeyer, para dirimir las discrepancias entre productores y compradores sobre los resultados de la polarización de los azucares crudos informados por los químicos sobre la misma muestra (Zerban, 1924).

En 1924, en una edición especial y separada, el LPSM, publicó The Reference Book of the Sugar Industry, e incluyó dos artículos directamente pertinentes al Caribe: "La agricultura y manufactura de la caña de azúcar en Jamaica”, por F. M. Kerr Jarret (1924), y "Las tierras vírgenes abandonadas en Cuba," de J. R. Zell (1924). En 1929, luego de 41 años de existencia, el LPSM se fusionó con otra revista azucarera, Facts about Sugar, que, a su vez, comenzó en 1914 y terminó en 1941.

\section{A MODO DE CONCLUSIÓN}

No deja de extrañar que las revistas azucareras, como el LPSM y otras mencionadas en este ensayo, no hayan sido más aprovechadas en investigaciones sobre las historias azucareras del Caribe, especialmente cuando en el Caribe hispanohablante, el renglón azucarero fue sustantivo de la segunda mitad del siglo XIX hasta por lo menos, la década de 1960 (Za- 
netti Lecuona, 2012). Se puede especular que, por su enfoque empresarial, su público se circunscribiera al de los negocios azucareros, por lo cual no se conocieron fuera de ese círculo mercantil. Además, por ser de ese mundo, es posible que no se les considere como fuentes confiables. A nuestro entender, esto último es poco probable. Si no lo fueran, su recepción hubiera sido agria en el mundo de la producción y mercadeo del dulce y no lo hubieran tomado en cuenta. Su durabilidad es evidencia de la confianza de su clientela ligada a la producción y la venta del azúcar en la gran mayoría de su contenido.

Las revistas azucareras han sido una fuente poco explorada que abren o complementan un amplio espectro de temas para diversos y variados estudios del azúcar en el Caribe, entre otros, de historia ambiental, económica, empresarial, tecnológica y social. Algunos estudios serían aquellos referentes a los circuitos (entrecruzados) imperiales del azúcar, la ciencia y tecno-

\section{NOTAS}

1 En 1899, el TSC se convirtió en 1899 en el International Sugar Cane Journal. No encontramos ningún trade journal azucarero en España, pero sí en Alemania. Por ejemplo, el Die Deutsche Zuckerindustrie, publicado en Berlín, Alemania (1879-1908).

2 Parte de los libros de la colección fueron adquiridos por la American Philosophical Society, también de Filadelfia, por los problemas económicos del Franklin Institute que vendió gran parte de su valiosa colección científica.

3 De estas sólo la cubana ha sido estudiada minuciosamente en Fernández, 2005

4 Los otros tres eran sus parientes Miguel Aldama y Alfonso, José Luis Alfonso García y Juan Poey Aloy.

\section{FUENTES PRIMARIAS}

A Barbadian (1897),"A Day on a Sugar Plantation in Barbados", Louisiana Planter and Sugar Manufacturer, 26 June, pp. 409412, 409 .

"Address" (1869), The Sugar Cane, 2 August, p. 1.

"A New Substitute for Sugar" (1893), Louisiana Planter and Sugar Manufacturer, 22 July, p. 147

"As to a Spanish Edition" (1911), Louisiana Planter and Sugar Manufacturer, 2 September, p. 147.

“Aviso" (1913), Louisiana Planter and Sugar Manufacturer, 5 July, p. 35. logía, la difusión del conocimiento práctico y científico y de innovaciones técnicas, el origen y evolución de los capitales azucareros, el desarrollo de las empresas individuales azucareras, el crecimiento de esta agroindustria en países particulares, los mercados de fuerza de trabajo, el papel de la esclavitud, las protestas y huelgas obreras, las migraciones, los asuntos gerenciales, la competencia entre países productores y entre países compradores, mercados nacionales y el mundial, el papel regulador de los estados, la competencia de azúcar de caña y de remolacha, el desarrollo de los sustitutos como la sacarina, el papel de los corredores de azúcar y las refinerías, la banca y los seguros, patentes y muchos otros. En suma, el LPSM ofrece un espectro de noticias y análisis útiles sobre los intereses involucrados directamente en la producción y mercadeo del azúcar de caña en el Caribe, y su consulta ofrece acceso a valiosa información usualmente de gran valor a los investigadores.

5 Además, en 1876, en su sección mensual de patentes, el TSC informaba que un tal "L. Z. Louve,", de Puerto Rico, solicitó una patente para "mejoras en la manufactura refinación de caña, remolacha, y otros azucares cristalizables" ("Monthly", 1876).

6 Dos años antes se pensó en publicar una "edición regular híbrida", parte en inglés y parte en español, pero fue descartada. El Mundo Azucarero tuvo una salida mensual de 1913 al 1939, luego trimestral de 1939 a 1945, y revirtió a mensual de 1946 en adelante hasta su cierre en 1956. Parece ser que esta publicación comercial también ha pasado inadvertida para los investigadores del azúcar aunque hay copia en el New York Public Library.

“Big Lillie Quadruple Effect for San Domingo". (1912), Louisiana Planter and Sugar Manufacturer, 2 March, p. 143.

Boyd, Overton F. (1924), "Sir Francis Watts - An Appreciation”, Louisiana Planter and Sugar Manufacturer, 16 May, pp. 392-393, 392.

Bovell, John R. (1911), "Report on a Visit to the Guanica Central Sugar Factory, Porto Rico", West Indian Bulletin, 11, pp. 56-59.

Browne, C. A, Dr. (1919), "Impressions of the Sugar and Syrup Industries in Barbados", Louisiana Planter and Sugar Manufacturer, 13 September, pp. 170-174.

Burton, Richard H. (1874), "A Visit to the First Central Sugar Factory Established in Porto Rico", The Sugar Cane, 1 August, p. 389. 
Burton, Richard H. (1875a), "Translation of a Report Made by Richard Henry Burton, Commissioned from Porto Rico to Visit the 'Usines' or Central Sugar Factories of Martinique, 1872", The Sugar Cane, 1 February, pp. 63-79.

Burton, Richard H. (1875b), "Translation of a Report Made by Richard Henry Burton, Commissioned from Porto Rico to Visit the 'Usines' or Central Sugar Factories of Martinique, 1872", The Sugar Cane, 1 March, pp. 119-136,

"Carl Wieting" (1919), Louisiana Planter and Sugar Manufacturer, 20 December, p. 396.

Carneiro Da Silva, J. José (1877), "The Industry of Sugar Making in the District of Quissama, Province of Rio de Janeiro, Brazil", The Sugar Cane, 1 December, p. 650.

"Central Factory in Jamaica" (1874), The Sugar Cane, 1 December, p. 236.

"A Change of Personnel at Our Havana Office" (1920), Louisiana Planter and Sugar Manufacturer, 4 December, p. 355.

Coombs, Frank (1893), “An American Observer in Cuba”, Louisiana Planter and Sugar Manufacturer, 22 March, pp. 242-245.

Coombs, Frank (1898), "Notes on the Sugar Industry of Trinidad, B.W.I.", Louisiana Planter and Sugar Manufacturer, 7 May, pp. 296-300.

Crawley, J. T. (1913), "Agricultural Developments in Porto Rico", Louisiana Planter and Sugar Manufacturer, 25 October, pp. 289-290.

"Cuba's Sugar Expansion and the Savannah Lands" (1914), Louisiana Planter and Sugar Manufacturer, 12 December, p. 372 .

Deerr, Nöel (1914a), "The Sugar Cane Industry", Louisiana Planter and Sugar Manufacturer, 12 December, pp. 380-383.

Deerr, Nöel (1914b), "The Sugar Cane Industry", Louisiana Planter and Sugar Manufacturer, 19 December, pp. 395-397.

Dominican (1913), "Santo Domingo", Louisiana Planter and Sugar Manufacturer, 18 November, p.43.

Dr. W. R. Dodson (1922), Louisiana Planter and Sugar Manufacturer, 1 April, p. 197.

Dr. F. W. Zerban (1922), Louisiana Planter and Sugar Manufacturer, 1 April, p. 197.

Edson, Hubert (1928), "Sugar Refining in Cuba", Louisiana Planter and Sugar Manufacturer, 28 July, pp. 62-63.

"Fisher Distributing Bagasse Burner" (1912), Louisiana Planter and Sugar Manufacturer, 27 July, p. 57.

Garnett, H. (1900), "San Domingo", Louisiana Planter and Sugar Manufacturer, 24 February, p. 123.

Granary, H. A. (1920), “Resume of the Development of New Factories, Changes in Ownership of Estates and Organization of New Sugar
Companies in Cuba during the Past Twelve Months", Louisiana Planter and Sugar Manufacturer, 4 September, pp. 155-158.

“John Dymond" (1922), Louisiana Planter and Sugar Manufacturer, 11 March, pp. 153-155.

"John S. Dennee to Represent the Louisiana Planter and El Mundo Azucarero in New York" (1919), Louisiana Planter and Sugar Manufacturer, 28 June, p. 277.

"Le Journal des Fabricants du Sucre, II y a 50 ans" (1865), Le Journal des Fabricants du Sucre, 12 de abril, p. 1.

"Journal de Fabricants de Sucre" (1919), Louisiana Planter and Sugar Manufacturer, 8 February, p. 95.

"Labor in Santo Domingo" (1919), Louisiana Planter and Sugar Manufacturer, 12 July, p. 29.

The Louisiana Planter and Sugar Manufacturer, disponible en: http://catalog.hathitrust.org/Record/000674886, [consultado el $12 / 1 / 2013]$.

“Louisiana Sugar News" (1918), Louisiana Planter and Sugar Manufacturer, 23 February, p. 120.

"Louisiana Sugar News" (1920), Louisiana Planter and Sugar Manufacturer, 24 January, p. 57.

Louisiana Planter and Sugar Manufacturer (1920), 13 January, p. 1.

"Major G. C. Comstock, Jr. Succeeds Mr. John S. Dennee as Manager of Our New York Office" (1920), Louisiana Planter and Sugar Manufacturer, 30 October, p. 503.

“Monthly List of Patents” (1976), The Sugar Cane, 1 May, p. 267

“New York” (1910), Louisiana Planter and Sugar Manufacturer, 19 August, p 117.

"Office of the Louisiana Planter in the City of Mexico, Avenue Isabel La Catolica, 72" (1914), Louisiana Planter and Sugar Manufacturer, 26 September, p. 206.

"Our Anniversary" (1904), Louisiana Planter and Sugar Manufacturer, 4 July, p. 7.

“Our Brazilian Office" (1921), Louisiana Planter and Sugar Manufacturer, 22 March, p. 226.

"Our Editor-in Chief" (1922), Louisiana Planter and Sugar Manufacturer, 3 June, p. 341.

“Our Havana Office” (1913), Louisiana Planter and Sugar Manufacturer, 8 February, p. 85.

“Our Havana Office" (1914a), Louisiana Planter and Sugar Manufacturer, 14 February, p. 98.

"Our Havana Office" (1914b), Louisiana Planter and Sugar Manufacture, 5 July, p. 3.

"The Outbreak of St. Croix" (1879), The Sugar Cane, 1 January, pp. 16-21. 
"Overton Boyd" (1957), Baton Rouge Morning Advocate, 27 November, p. 7.

Palmer, Truman G. (1918), “Dr. Lewis S. Ware, June 18, 1851-Dec. 20, 1918", Louisiana Planter and Sugar Manufacturer, 2 August, pp. $89,93-95$

"Personal" (1912), Louisiana Planter and Sugar Manufacturer, 4 August, p. 123.

Prinsen Geerligs, H. C. (1929), “Cane Sugar, pt. I", Louisiana Planter and Sugar Manufacturer, 15 June, pp. 461-463, 480.

Prinsen Geerligs, H. C. (1929), “Cane Sugar, pt. II”, Louisiana Planter and Sugar Manufacturer, 22 June, pp. 481-482.

"Private Advice's from Cuba" (1893), Louisiana Planter and Sugar Manufacturer, 4 March, p. 181

"The Redivivus of Hayti, the Black Republic" (1916), Louisiana Planter and Sugar Manufacturer, 16 July, p. 36.

Rolfe, George W. (1912), "Some Notes on Sugar Manufacture in Porto Rico", Louisiana Planter and Sugar Manufacturer, 26 October, pp. 283-285.

Ruegg, S. G. (1922a), "Historical Sketch of Glasgow Sugar Machinery", Louisiana Planter and Sugar Manufacturer, 5 August, pp. 89-90.

Ruegg, S. G. (1922b), “A Noted Scotch Machinery House”, Louisiana Planter and Sugar Manufacturer, 16 September, pp. 183-184.

Ruegg, S. G. (1922c), "Fawcett, Preston \& Co., Ltd., of Liverpool", Louisiana Planter and Sugar Manufacturer, 4 November, pp. 303-304.

Sagra, Ramón de la (1865a), "Sur les ameliorátions a apporter dans L'ile de Cuba", Le Journal des Fabricants de Sucre, 26 de enero, pp. 1-2.

Sagra, Ramón de la (1865b), “Correspondance”, Le Journal des Fabricants de Sucre, 26 de enero, p. 2

Sagra, Ramón de la (1865c), "Sur les ameliorátions a apporter dans L'ile de Cuba (fin)", Le Journal des Fabricants de Sucre, 9 de febrero, pp. 1-2.

\section{BIBLIOGRAFÍA}

Bass, William L (1902), Reciprocidad. Exposición presentada al gobierno de la República Dominicana, Santo Domingo, Imp. Cuna de América.

Beachey, R. W. (1957), The British West Indies Sugar Industry in the 19th Century, Oxford, Blackwell.

Brand, Robert F. (1948), "Spanish in American Trade Journals", The Modern Language Journal, 32 (6) October, pp. 436-441.

Commodity Histories, disponible en: http://www. commodityhistories.org, [consultado el 7/1/2014].
Sagra, Ramón de la (1867), "Correspondance", Le Journal des Fabricants de Sucre, 10 de enero, p. 1.

"Santo Domingo" (1892), The Sugar Cane, 1 July, p. 362.

"The Shops of Manuel Galdo \& Co." (1917), Louisiana Planter and Sugar Manufacturer, 1 September, pp. 139-141.

"Situations Wanted" (1905). Louisiana Planter and Sugar Manufacture, 7 January, p. 16.

Spencer, Guilford L. (1923), "Chemical Progress in Cane Sugar Manufacture", Louisiana Planter and Sugar Manufacture, 13 January, pp. 32-33.

"Standard Granulated Sugar of Superior Quality Made at Central Mercedita, Porto Rico" (1926), Louisiana Planter and Sugar Manufacturer, 2 July, p. 15.

"Sugar Market to be Broadcasted by Radio" (1924), Louisiana Planter and Sugar Manufacturer, 29 March, p. 247.

The Sugar Cane, disponible en: http://babel.hathitrust.org/cgi/ pt?id=nyp.33433008135281, [consultado el 22/1/2014].

The Sugar Cane (1902), February, p. 61.

Thomson, D. L. (1904), "Sugar in Porto Rico and Guanica Central Company", Louisiana Planter and Sugar Manufacturer, 2 April, pp. 238-239.

"Thos. D. Boyd, Jr. Comes to Havana" (1922), Louisiana Planter and Sugar Manufacturer, 14 October, p. 271.

Timehri, disponible en: https://archive.org/details/timehrijournalr06guiagoog, [consultado el 8/3/2014]

"Twenty-Five Years of Sugar Journalism" (1913), Louisiana Planter and Sugar Manufacturer, 6 July, pp. 9-14.

Weinrich, Moriz [Maurice] (1881), "The Sugar Industry in Cuba, its Financial Embarrassments, the Cost of the Price of Sugar", The Sugar Cane, 1 July, pp. 357-361.

Wieting \& Richter (1905), "British Guiana", Louisiana Planter and Sugar Manufacturer, 7 January, p. 10.

Crespo, Horacio (2006), "Trade Regimes and International Sugar Market, 1850-1980: Protectionism, Subsidies, and Regulation", En: S. Topik, C. Marichal \& Z. Frank (eds.), From Silver to Cocaine: Latin American Commodity Chains and the Building of the World Economy, 1500-2000, Durham, Duke University Press, pp. 147-203.

Fernández Prieto, Leida (2005), Cuba agrícola: mito y tradición, 18781920, Madrid, Consejo Superior de Investigaciones Científicas.

García Muñiz, Humberto (2010), Sugar and Power in the Caribbean: The South Porto Rico Sugar Company in Puerto Rico and the 
Dominican Republic, 1900-1921, San Juan, Kingston, Editorial de la Universidad de Puerto Rico, Ian Randle Publishers,, pp. 189211. Hay traducción al español por la Academia Dominicana de la Historia, 2014.

Heitmann, John Alfred (1987), The Modernization of the Louisiana Sugar Industry 1830-1910, Baton Rouge, Louisiana State University Press.

Iglesias García, Fe (1998), Del ingenio al central, San Juan, Editorial de la Universidad de Puerto Rico.

Kerr Jarrett, F. M. C. (1924), "Sugar Cane Agriculture and Manufacture in Jamaica", En: The Reference Book of the Sugar Industry in the World, New Orleans, Louisiana Planter and Sugar Manufacturer, Co., Inc., pp. 50-51.

McAvoy. Muriel (2003), Sugar Baron: Manuel Rionda and the Fortunes of Pre-Castro Cuba, Gainesville, University Press of Florida.

Moreno Fraginals, Manuel (1986), El ingenio: complejo económico social cubano del azúcar, vol. 3., La Habana, Editorial de las Ciencias Sociales, p. 234.

Palmer, Truman G., (comp.) (1914), Translations from European Sugar Journals, Issues of 1911-12 and 1913, Wash., D.C., U.S. Beet Sugar Industry, p. 10.

Rados, Carol (2003), "Former FDA Press Officer Looks Back at 100", FDA Consumer, October-December, disponible en: http:// permanent.access.gpo.gov/Ips1609/www.fda.gov/fdac/ features/2003/503_emil.html, [consultado 12/7/2013].

Sagra, Ramón de la (1838-1857), Historia física, política y natural de la isla de Cuba, Paris, A. Bertrand.
Sawa, Bozena (2014), "New Yorkers! StopDemolition ofHistoric Domino Sugar Refinery in Brooklyn", disponible en: https://www.linkedin. com/today/post/article/20140707170428-217942005-new-yorkers-stop-demolition-of-historic-domino-sugar-refinery-in-brooklyn, [consultado el 16/7/2014].

Schnakenbourg, Christian (1984), "From Sugar Estate to Central Factory: The Industrial Revolution in the Caribbean", En: Bill Albert y Adrian Graves (eds.), Crisis and Change in the International Sugar Economy 1860-1914, Norwich, ISC Press, pp. 83-94.

Talbot, John M. (2002), "Tropical Commodity Chains, Forward Integration Strategies and International Inequality: Coffee, Cocoa and Tea", Review of International Political Economy, disponible en: http://www.tandf.co.uk, DOI: 10.1080/0969229022000021862.

The Reference Book of the Sugar Industry in the World (1924), New Orleans, Louisiana Planter and Sugar Manufacturer, Co., Inc.

Zanetti Lecuona, Oscar; Hernán Venegas Delgado, y Humberto García Muñiz (2001), "Nöel Deerr en la Guayana Británica, Cuba y Puerto Rico, 1897-1921", Revista Mexicana del Caribe, 6 (11), pp. 57-154

Zanetti Lecuona, Oscar (2102), Esplendor y decadencia del azúcar en las Antillas hispanas. La Habana, Editorial Ciencias Sociales y Casa Editorial Ruth.

Zell, J. R. (1924), "Abandoned Virgin Soils in Cuba”, En: The Reference Book of the Sugar Industry in the World, New Orleans, Louisiana Planter and Sugar Manufacturer, Co., Inc., pp. 71-74.

Zerban, F. W. (1924), "The New York Sugar Trade Laboratory", En: The Reference Book of the Sugar Industry in the World, New Orleans, Louisiana Planter and Sugar Manufacturer, Co., Inc., pp. 41-43. 